\title{
A dietary intervention with Akkermansia muciniphila and quercetin supplementation reshapes gut microbiota composition in an in vivo model of early obesity related non-alcoholic fatty liver disease
}

\author{
M. Juárez-Fernández ${ }^{1}$, D. Porras ${ }^{1}$, P. Petrov $^{2}$, M.V. García-Mediavilla ${ }^{1,3}$, S. Román Sagüillo ${ }^{1}$, \\ S. Martínez-Flórez ${ }^{1}$, J. González-Gallego ${ }^{1,3}$, E. Nistal ${ }^{1,3}$, R. Jover ${ }^{2,3}$ and S. Sánchez-Campos ${ }^{1,3}$ \\ ${ }^{1}$ Instituto Universitario de Biomedicina (IBIOMED), Universidad de León, Spain, \\ ${ }^{2}$ Unidad de Hepatología Experimental, IIS Hospital La Fe, Valencia, Spain and \\ ${ }^{3}$ Centro de Investigación Biomédica en Red de Enfermedades Hepáticas y Digestivas (CIBERehd), Instituto de Salud \\ Carlos III, Madrid, Spain
}

Gut microbiota modulation by nutritional procedures has become a feasible therapeutic target in the management of obesity and nonalcoholic fatty liver disease (NAFLD) ${ }^{(1)}$. Recently, Akkermansia muciniphila has been considered as a potential probiotic due to its protective effect in obesity development ${ }^{(2)}$. Besides, the flavonoid quercetin has shown an anti-inflammatory, antioxidant and prebiotic capacity, highlighting a great therapeutic potential in NAFLD ${ }^{(3)}$. This study aims to evaluate the effect of gut microbiota modulation by the feasible symbiotic quercetin and A. muciniphila combined with nutritional intervention in the development of early obesity-related NAFLD in an in vivo model.

21-days old Wistar rats were fed with a high-fat diet (HFD) or a control (C) diet for 6 weeks. Afterwards, all animals were fed with a $\mathrm{C}$ diet supplemented or not with quercetin and A. muciniphila (administered daily by oral gavage) for 3 weeks. Faecal samples were analysed by qPCR and sequenced by Illumina MiSeq System to determine gut microbiota composition. Statistical significance was determined by nonparametric Kruskal-Wallis test followed by Mann-Whitney U test $(p<0.05)$.

After 6 weeks, HFD intake triggered a lower concentration of the total bacteria DNA in comparison to C, but increased gut microbiota diversity calculated by the Shannon diversity index. Metagenomic analysis demonstrated an HFD-related gut dysbiosis, showing a significant increase of Firmicutes, Proteobacteria and Actinobacteria phyla, while, Bacteroidetes and Cyanobacteria showed an opposite pattern. At genus level, HFD increased the relative abundance of Adlercreutzia, Allobaculum, Bifidobacterium, Bilophila, Blautia, Clostridium, Enterococcus, Lactobacillus, Oscillospira, Rothia and Streptococcus genera. In contrast, Coprobacillus, Coprococcus, Phascolartobacterium, Prevotella, Roseburia, Sutturella and Turicibacter were reduced. Principal coordinates analysis (PCoA) revealed that the bacterial communities of HFD-fed rats clustered separately from those of C group. After 3 weeks, the nutritional intervention together with quercetin and A. muciniphila supplementation reversed the gut dysbiosis associated to HFD, tending to increase total bacteria counts and decrease Shannon diversity index. Moreover, a notable increase in the abundance of Cyanobacteria phylum, as well as a decrease in Actinobacteria phylum was detected. Additionally, several differences at genus level were observed in the symbiotic supplemented groups that were not detected with the administration of any of the treatments alone. The symbiotic reduced the detection of Lactococcus and Lactobacillus genera, whereas Oscillospira was highly abundant in both supplemented groups. To determine the effect of quercetin and A. muciniphila administration on bacterial communities a PCoA was conducted, showing that the individuals receiving the symbiotic tended to cluster together and separated from the other groups.

Dietary intervention combined with A. muciniphila and quercetin administration seems to counteract HFD-associated dysbiosis and subsequent obesity related-NAFLD in a juvenile rat model.

\section{Acknowledgements}

Supported by BFU2017-87960-R; GRS1888/A/18. CIBERehd is funded by Instituto de Salud Carlos III. DP and SRS are supported by a fellowship from Junta de Castilla y León, co-financed by the European Social Fund. MJF is supported by a fellowship from the Ministry of Education of Spain (FPU18/06257). E.N. was supported by CIBERehd contracts.

\section{References}

1. Polyzos SA, Kountouras J \& Mantzoros CS (2019) Metabolism 92, 82-97.

2. Depommier C, Everard A, Druart C et al. (2019) Nat Med 25, 1096-103.

3. Porras D, Nistal E, Martínez-Flórez S et al. (2019) Mol Nutr Food Res 63. 